Review article

Paediatrics Today 2016;12(1):30-40

DOI $10.5457 / \mathrm{p} 2005-114.133$

\title{
THE ROLE OF CONVENTIONAL ULTRASOUND IN PEDIATRIC CHRONIC LIVER DISEASES
}

\author{
Alemka JAKLIN KEKEZ*, Andrea CVITKOVIĆ ROIĆ, Iva PALČIĆ \\ Polyclinic for pediatric disease \\ Helena, Zagreb, Croatia \\ *Corresponding author: \\ alemkajk@gmail.com \\ Tel.: + 38512300167 \\ Fax.: + 38512310197 \\ Received: November 22, 2015 \\ Accepted: January 3, 2016 \\ The aim of this article is to provide an overview of conventional ul- \\ trasound in imaging chronic liver diseases in children. The first part \\ describes the normal ultrasound appearance of a child's liver and the \\ concepts of examination. The further sections present the usual ul- \\ trasound findings, the key sonographic points of the most common \\ chronic paediatric liver conditions, and the role of ultrasound in pro- \\ viding diagnostic and prognostic information, detecting complica- \\ tions and supporting reassuring liver biopsy. The limitations of the \\ method are also discussed. Taking all this into account, the interpreta- \\ tion of ultrasound is better in the context of the clinical picture and, \\ in inconclusive cases, followed up with additional imaging and labora- \\ tory work up. Conclusion - In conclusion, regardless of the progress \\ made in diagnostic methodology, ultrasound still remains the first, but \\ mostly not the last imaging modality for presumed chronic liver dis- \\ Key words: Ultrasound - Liver - Children. \\ eases, in daily clinical practice.
}

\section{Introduction}

The liver is the central metabolic organ in the body, included in many essential functions: digestion, metabolism, storage of nutrients, detoxification and immunity. It has a very large functional reserve and reparative potential. Therefore, it may suffer extensive damage before malfunction becomes clinically significant and apparent. This is the reason why some pathological conditions may stay hidden for a long period of time. Fortunately, simple biochemical liver tests and noninvasive methods, such as ultrasound (US), can reveal liver pathology before the appearance of advanced clinical signs. On the other hand, in the case of clinically suspected liver disease, US can provide valuable information about the liver, the extent and type of pathology, and guide the further diagnostic work up. There are also many other wellknown advantages of the US technique. It is a non-invasive, non-ionizing, painless, safe, and comparatively cheap method, which can be performed without complicated preparation. As the image is captured in real-time, it can show the structure and movement of the body`s internal organs. However, US also has some limitations which are discussed below $(1,2)$.

The aim of this review is to present arguments as to why conventional US is still regarded as the optimal first line imaging technique for presumed liver disease, prior to other more advanced techniques, as well as the function of ultrasound in diagnosing specific liver conditions and further monitoring. 


\section{The ultrasound approach - general concept}

The US approach, in suspected liver disease, should be systematic and include the examination not only of the liver, but also of other abdominal organs. The goal is to cover the entire abdomen, including the pelvis, looking for associated conditions and complications of liver disease, such as ascites or signs of portal hypertension (changes to the portal vein calibre, collaterals, portosystemic shunts, reversed portal flow, splenomegaly) (3). The spleen length is best to be compared with the adjacent kidney`s length, as they are normally the same size. A good overview of the liver itself means analysing its size and shape, parenchyma, the gallbladder, the bile ducts and blood vessels, as well as searching for focal lesions. Liver size in children should be evaluated by measuring the length in medioclavicular, sternal and anterior axillary planes, in comparison with standards adapted to the child's height $(4,5)$. The shape of a healthy liver is pyramidal, with the exception of a sometimes visible Riedel lobe. This is the common, anatomical, tongue-like protuberance of the right liver lobe (6). The hepatic margins are normally smooth and sharp. Falciform ligament divides the parenchyma into the left and right lobes, each of which has a well-defined segmental structure. A basic knowledge of liver anatomy and segment borders is essential for describing the precise positioning of liver pathology, especially the focal changes. The normal parenchymal texture is homogenous, and of medium echogenicity, slightly brighter than the renal cortex, and slightly darker than the spleen. In early infancy, due to the increased echogenicity of the renal cortex, the difference is minimal. Changes in echogenicity always suggest a liver parenchymal disorder. However, normal echogenicity cannot completely exclude a mild pathological condition. Sonographic examination of the liver should include visu- alization of the gallbladder and the biliary tree. They are best examined after a 4 to 6 hour fast. In this way, the normal gallbladder will be full of bile and distended, which makes examination optimal. Its length should not exceed that of the nearby kidney. The normal wall thickness of the distended gallbladder in children is up to $3 \mathrm{~mm}$. Finally, it should be evaluated for the presence of gallstones, inspissated bile and thickened wall. The intrahepatic bile ducts are ordinarily not visible, whereas when dilated they typically produce a 'double barrel shotgun' appearance. The common bile duct (CBD) should not exceed a calibre of $1 \mathrm{~mm}$ in neonates, $2 \mathrm{~mm}$ in infants, $4 \mathrm{~mm}$ in older children. Careful examination of the blood vessels is also an integral part of liver US, routinely combined with Doppler. Hepatic vascularisation is rich and complex, with a large number of variations. It is supplied by the hepatic artery and portal vein, and venous drainage is handled by hepatic veins. On sonograph, the peripheral portal venous vasculature and hepatic artery are plainly demarcated, with bright visible walls, whereas the edges of the hepatic veins are thin and hypoechoic, and therefore not accentuated. Measurement of the portal vein diameter is helpful for diagnosis of portal hypertension. The mean diameter is $8.5 \mathrm{~mm}$ in children younger than 10 years of age and 10 $\mathrm{mm}$ in older ones. Doppler is primarily used to determine the presence and direction of the portal flow and its velocity, to check the spectrum of the hepatic veins and also to measure their flow velocity, as well as to evaluate the flow and resistive index in the hepatic artery. Furthermore, it is helpful in differentiating blood vessels from bile ducts, if there is any doubt about this. The last, but not the least use is searching for enlarged (wider than $5 \mathrm{~mm}$ ) lymph nodes in the liver hilum and the hepatoduodenal ligament $(3,7,8)$.

The limitation of US examination is that US waves may be disrupted by gas or subcutaneous fat in obese patients, or some other 
unfavourable effects, such as reverberation. Therefore, some parts of the abdominal cavity or organs may remain obscured. Some areas of the liver are also more difficult to examine, such as the superficial liver above the costal margins, the left tip of the lateral segment of the left lobe, and the ventral subdiaphragmatic regions. Further, another limitation of US is that different pathologies may have similar nonspecific findings, and the interpretation depends on the expertise of the operator. To reduce error, it is always important to follow the same routine for examinations, to use different available approaches and optimal transducers, and to be aware of the limitations of US $(4,9)$.

\section{Fatty liver}

Non-alcoholic fatty liver disease (NAFLD) covers a spectrum of conditions, ranging from a simple fatty infiltration (steatosis) to non-alcoholic steatohepatitis (NASH), characterized by inflammation and sometimes fibrosis. In developed countries, liver steatosis and NASH are becoming one of the common causes of paediatric chronic liver disorders today. In adults, the pattern of inflammation is usually lobular, and of fibrosis perivenular (Type $1 \mathrm{NASH}$ ), whereas in children it is periportal (type $2 \mathrm{NASH})(10,11)$. The inflammation and degree of fibrosis are the key prognostic parameters for advanced liver disease, which later on, usually in adulthood, may progress to cirrhosis (12). Besides NAFLD, fatty accumulation in the liver may be part of different conditions, such as: cystic fibrosis, parenteral nutrition associated liver disease, malnutrition, some metabolic liver diseases such as Wilson, or may be toxic, such as drug induced liver disease (10).

Ultrasonographically, fat accumulation in the liver is represented by increased parenchymal echogenicity (bright liver) and poor distinction of the blood vessel borders and diaphragm due to decreased acoustic pen- etration (10). Grading is usually evaluated as mild (grade 1), moderate (grade 2) or severe (grade 3). Grade 1 is represented by a slight increase in the echoes of the parenchyma, with normal visualisation of the vessel wall and the diaphragm. Grade 2 is represented by a higher increase in hepatic echogenicity and slightly impaired visualisation of the intrahepatic vessels and the diaphragm. Grade 3 means marked reinforcement of brightness with indistinct vessel wall and diaphragm (13). The usual distribution is diffuse, in rare cases (in grades 2 and 3) with subtle multifocal sparing. Less common patterns include diffuse heterogeneous deposition, and focal or multifocal deposition (Fig. 1).

These last two may mimic neoplasm, and require more sensitive imaging procedures such as MRI (14). In adults, the sensitivity of US for fatty liver detection seems to be around 60 to $94 \%$ and its specificity about 66 to $95 \%$ (15). In children, data analysing its accuracy are scarce. A systematic review conducted in 2014 , based on the available studies in children, shows the positive predictive value of US to be between 47 to $62 \%$ in detecting and grading fatty liver in children. The sensitivity for revealing mild steatosis in adults is lower than for other grades, whereas high quality studies are lacking in children (16). Fibrosis and inflammation may also intensify liver echogenicity, but they are associated with liver enlargement more often than simple steatosis. Nevertheless, these findings all together are not reliable enough to distinguish steatosis from fibrosis, particularly initially. So, objective quantification of steatosis and its differentiation from $\mathrm{NASH}$, mild fibrotic or fatty fibrotic liver are not possible by conventional US alone. Sensitivity and specificity are additionally decreased in very obese children (17). As many other different clinical conditions may also present with fatty liver, differentiation between them should be made only by combining clinical, biochemical, imaging and, finally, histology work ups $(10,11,15)$. 


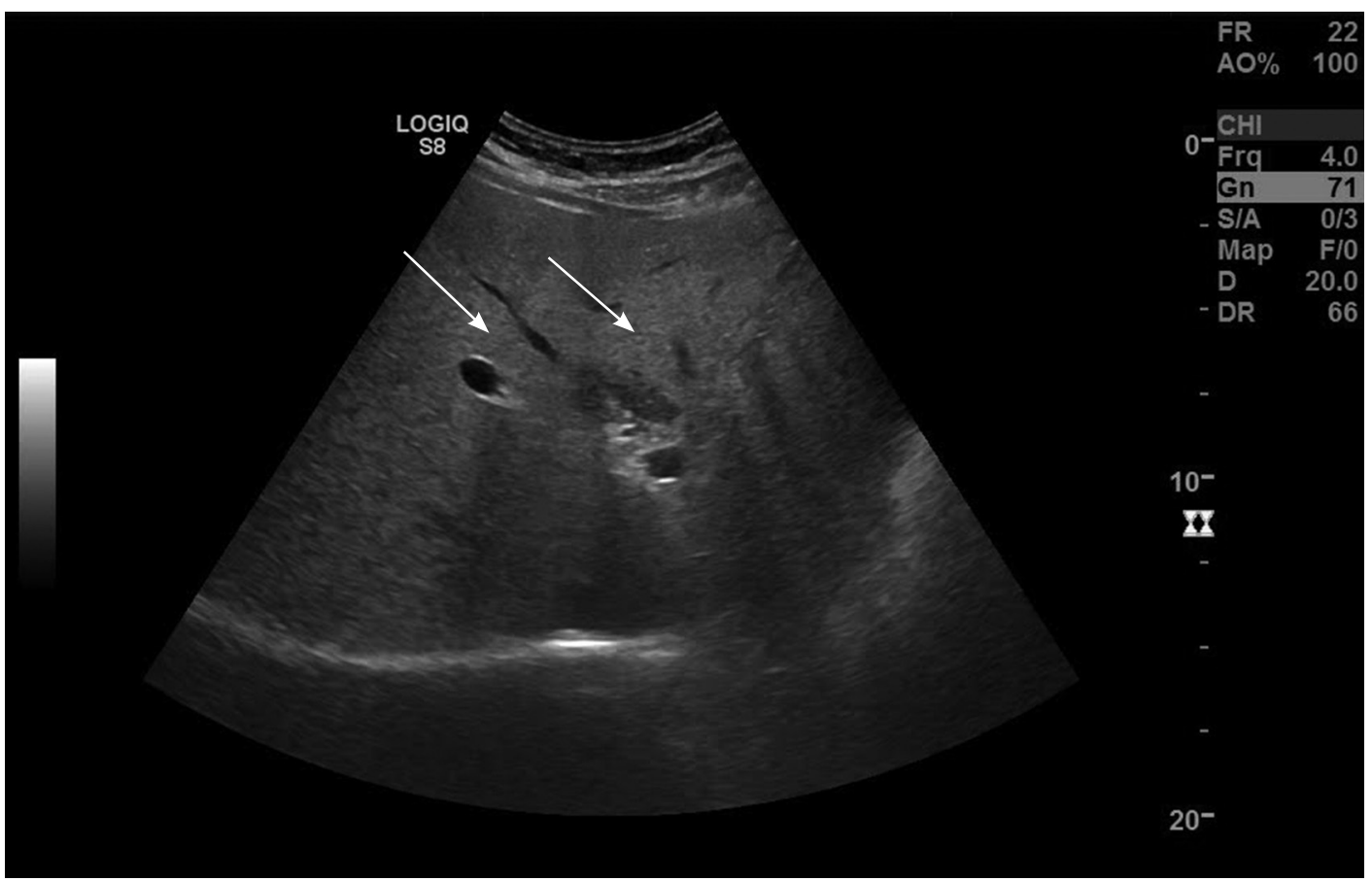

Fig. 1 Ultrasound of a child with steatosis areata: multifocal hyperechoic fat deposition in the right lobe of the liver.

\section{Fibrosis and cirrhosis}

Chronic liver disease (CLD) in children encompasses a broad spectrum of conditions that vary with the age of appearance. The clinical presentations are commonly very similar, which makes correct diagnosing more difficult. In infancy and early childhood, the main causes of CLD are biliary atresia (BA) and neonatal hepatitis due to metabolic diseases (alpha-one antitrypsin deficiency, galactosemia, etc.), infections (viral, bacterial and parasitic) or diseases of unknown aetiology (idiopathic). In older children, the more common forms are Wilson disease, autoimmune hepatitis (AIH) or sclerosing cholangitis (ASC), NASH, chronic viral hepatitis, post Kasai status, and cystic fibrosis. Regardless of aetiology, the development of fibrosis is an unavoidable part of the natural history of most CLDs. Moreover, it may progress to cirrhosis, which is an advanced stage of fibrosis, accompanied by distortion of the hepatic vasculature and liver architecture. Cirrho- sis in children tends to present with a silent clinical course, followed by the onset of liver dysfunction and portal hypertension signs. The most important predictor of decompensation is an increase in intrahepatic venous resistance and portal blood flow, representing portal hypertension (18). Studies which have analysed some of the previously listed CLDs in children have shown quite advanced fibrosis and/or cirrhosis, even during the establishment of the diagnosis: in $69 \%$ of those with AIH type 1, 38\% in AIH type 2, 21\% in patients with Wilson disease, $25 \%$ in chronic $\mathrm{HCV}$ infection, $3.5 \%$ in chronic HBV and about 3\% in NAFLD (19). Recent developments in the understanding of liver fibrogenesis have revealed that the process is dynamic and may be partially reversed from any degree of fibrosis, including those associated with cirrhosis. Current advances in diagnostic and treatment procedures enable better management of CLD and currently significantly contribute to longer life expectancy (20). 
US is a regular screening tool for fibrosis and cirrhosis in CLD. Sonographic findings of fibrosis include echogenic liver, with a mildly to highly coarse and heterogeneous echotexture. Compared to steatosis, fibrosis does not increase the beam attenuation. Increased echogenicity around the portal veins may indicate periportal fibrosis, as in congenital hepatic fibrosis (5). The fibrotic liver may be of different sizes, which mostly depends on the progression of the liver disease; when moving towards cirrhosis it becomes smaller, sometimes with compensatory hypertrophy of the left or /and caudate lobe. The surface becomes irregular with nodularity of the margins. Eventually, a shrunken liver is a positive sign of cirrhosis. The cirrhotic parenchyma shows inhomogeneity and coarseness, with commonly indistinct intrahepatic vessels, which can be difficult to distinguish from fibrosis. The persistence of hypoechoic areas, as the result of regenerating nodules, points more to cirrhosis. Rarely, parenchymal nodularity may be seen in steatosis. Taken together, when compared to the echo-texture, liver surface nodularity has greater significance for the presence of cirrhosis. Detection of ascites and splenomegaly, and/or signs of portal hypertension (varices, collateral venous channels, spontaneous portosystemic shunts, and retrograde flow in the portal vein) further highlight diagnosis. Studies in adults demonstrate the high accuracy of US (over 90\%) in confirming the diagnosis of cirrhosis in patients with complications. Early stages and micronodular cirrhosis may be overlooked in up to $30 \%$ of cases $(9,21)$. Such data are missing in children.

Shear wave elastography (SWE) is a relatively new technique which has been shown as a more accurate method for liver fibrosis staging than conventional US. However, precise differentiation of fibrosis stages cannot be achieved even with SWE $(22,23)$. Further on, we will represent the most useful sonographic findings in specific CLDs in children.

\section{Neonatal and infant chronic liver disease}

In the neonatal period and infancy, liver disease is mostly presented with persistent conjugated jaundice, while the aetiology is usually not easy to define. However, the priority for a good outcome is promptly to allocate the potentially treatable conditions, such as BA, choledochal cyst and some metabolic disorders, before they progress to end stage liver disease. Therefore, early diagnosis is a challenge for all paediatric hepatologists. The first imaging of a jaundiced baby, standardly made by US, may show a spectrum of changes: some that are not specific and overlap within diseases, some that are more specific that may predict the aetiology, or those that are very specific and sufficient for ultimate diagnosis $(3,24)$.

US is the examination of choice for detecting choledochal malformation and bile sludge. It has been shown to be superior to computed tomography in identifying this pathology, and it avoids unnecessary radiation. The typical US finding of the most common malformation (typical choledochal cyst) is fusiform or a round anechogenic zone in the liver hilus, separate from the gallbladder, representing dilatation of the CBD. Dilatation of the hepatic and intrahepatic ducts may or may not be seen. About 25\% of choledochal cysts present in the neonatal period, while up to $15 \%$ are even detected antenatally. The widely accepted classification sorts choledochal cysts into five different subtypes, based on the site of dilatation. Precise identification by US depends on the skill of the operator. Before a surgical decision, detailed information about the biliary tree is necessary, as well as the complete exclusion of all the conditions in the differential diagnosis $(25,26)$. US image sensitivity is about 71 to $97 \%$. It decreases in the presence of overlying bowel gas, pancreatitis or cholangitis, and may be misinterpreted and confused with 
pancreatic pseudocysts, hepatic, mesenteric cysts, acute fluid collection and most often with cystic biliary atresia (CBA). CBA is a rare form of $\mathrm{BA}$, characterised by a cyst in an obliterated biliary tree, and is mostly indistinguishable from a choledochal cyst by US alone. However, ultrasonography, followed by MRCP or cholangiography in most cases provides sufficient information for preoperative surgical evaluation of the whole biliary tree, and for clarifying doubtful cases. For CBA, liver biopsy is the gold standard. US is the preferred investigation in postoperative surveillance $(26,27)$. Furthermore, a recent study has demonstrated the high diagnostic value of the US (with multislice views and good reproducibility) in the preoperative diagnosis of paediatric choledochal cyst perforation. The ultrasound findings significantly correlated to the surgical findings (28).

Echosonography is less useful for differentiating BA from neonatal hepatitis, but is still a good first imaging method. In both conditions, liver size may be either normal or enlarged, and the parencyhmal echogenicity is usually increased and more or less inhomogeneous. An absent, small or abnormal gallbladder, after an adequate fast, is more suggestive of BA, while a preserved one suggests neonatal hepatitis. Nevertheless, if the atretic CBD is distal to the insertion of the cystic duct, a normal-sized gallbladder may be seen in BA. Also, a shrunken one may be seen in neonatal hepatitis. Changes to the gallbladder after a meal imply patency of the bile ducts, whereas their absence is more indicative of a BA. Due to the small calibre at that age, the CBD duct may be difficult to find, even in healthy infants. Due to improved US technology, good evaluation of CBD is possible, as well as verification of the patency of smaller bile ducts (4). The most specific US finding for BA is the so-called 'triangular cord' (TC). This is a tubular echogenic cord of fibrous tissue seen in the porta hepatis, which represents the obliterated duct remnant. The usual US criteria for the TC sign is thickness of the echogenic anterior wall of the right portal vein larger than $4 \mathrm{~mm}$, measured by an experienced ultrasonographer (29). A careful search should also be made for other abnormalities in the abdomen, such as: polysplenia, asplenia, preduodenal portal vein, interrupted inferior vena cava, and situs inversus. All of these may be associated with BA. In summary, none of the aforementioned US signs are sufficiently reliable alone. Therefore, US should not be the sole procedure used on which the differentiation is based (30). A recent study evaluated the competence of high resolution ultrasound (HUS) instead of the conventional one. The results showed better sensitivity, specificity, and accuracy, 91.3\%, $92.9 \%$, and $92.2 \%$, respectively, for the diagnosis of BA. It was concluded that HUS provided better imaging than conventional US, although the limitation of this study is the small number of cases (31).

Inspissited bile syndrome is a rare but not negligible cause of conjugated jaundice in small children. It is characterized by viscid or/and thick bile due to various reasons: massive haemolysis, increased enterohepatic circulation, cystic fibrosis, total parenteral nutrition, prematurity, diuretic therapy, etc. The diagnosis is usually obvious on US. Images commonly demonstrate a bile plug with echogenic content in the gallbladder, while the absence of shadowing distinguishes it from calculi. The CBD is usually dilated, filled with sludge, with small bile ducts secondary dilated (26).

\section{Chronic liver disease in older children}

As previously mentioned, autoimmune hepatitis or/and sclerosing cholangitis, Wilson disease, NAFLD, alpha 1 antitrypsin deficiency, chronic viral hepatitis and liver disease in cystic fibrosis generate the majority of 
chronic liver conditions in older children. As at a younger age, detecting the aetiology may be a matter of survival in potentially treatable diseases (autoimmune, Wilson, NAFLD), while the evaluation of severity plays an important role in following up and predicting the outcome. A significant clinical problem is that these conditions are generally nonspecific in their presentation, as are the results of basic liver tests. Unfortunately, the US appearance is also predominantly nonspecific. It typically shows hyperchoic liver parenchyma, with greater or lesser coarseness, and an enlarged or decreased liver size, depending on the severity of the underlying process. Hence, it may be primarily helpful in confirming the chronic nature of the disease, and discovering more advanced stages. Less often, US scans confidently indicate a specific disorder. However, neither CT nor MRI is superior to ultrasonography in providing available information about hepatic parenchyma, or directing the diagnosis in patients with CLD. The exceptions are biliary changes, where MRCP is much more helpful $(2,32)$.

The presence of enlarged lymph nodes of the hepatoduodenal ligament, in addition to signs of a chronic hepatobiliary disease, may suggest an autoimmune aetiology or chronic viral hepatitis. Interestingly, perihepatic lymphadenopathy has never been found in metabolic or toxic liver damage (33).

The diagnosis of AIH and ASC is based on a series of inclusion and exclusion criteria, which do not include US appearance. However, US may be helpful in differentiating AIH from ASC or for detecting the overlap syndrome, which is significantly more common in children than in adults. It may show ductal wall thickening, focal bile duct dilations, or stricturing of the CBD. Other findings may include gallbladder wall thickening, distension, and gallstones. US does not have high sensitivity for clarifying these conditions, especially for a specific subgroup of ASC
- 'small-duct disease'. In this type, which is rarely reported in the paediatric population, US findings, and even those of the MRCP, may be normal, without evidence of biliary involvement. In a study where cholangiography (predominantly MRCP) results were compared to histology findings, biliary diseases were missed in a significant proportion (36\%) of radiological findings. However, a quarter of the children with ASC, based on abnormal cholangiograms, had no histological features of bile duct involvement. Therefore, hepatologists should be very careful in making conclusions $(34,35)$.

In Wilson disease, US may only show signs of fatty infiltration or mild fibrosis, particularly in early parenchymal alterations, which are more common in children. Furthermore, US has not been proposed as a part of the diagnostic algorithm for confirming the diagnosis of Wilson`s disease $(36,37)$.

For patients with CF, there is a spectrum of possible hepatobiliary changes termed 'CF-associated liver disease' (CFLD). The average age of CFLD diagnosis is 10 to 11 years, and more than $90 \%$ are diagnosed before the child reaches their twenties. Clinical manifestations are usually inconspicuous and only 40 to $50 \%$ of children with CF exhibit intermittent elevation of liver tests. Furthermore, biochemical markers may not reliably identify patients with CFLD, nor predict progression to end-stage liver disease. Fortunately, hepatocellular function usually remains well preserved for years. An US finding of an altered hepatobiliary system frequently precedes clinical presentation and abnormal biochemical liver tests, and is therefore more sensitive for the diagnosis of CFLD. One of the most common early lesions is steatosis. The most typical liver features of CF are focal biliary cirrhosis (FBC) and multilobular cirrhosis, with or without portal hypertension. On US, FBC is characterized by a larger than $2 \mathrm{~mm}$ thickness of the hyperechoic periportal 
tissue. The usual appearance of the less frequent multilobular cirrhosis is an irregular, nodular liver and coarse heterogeneous parenchyma. Longitudinal studies found that only 20 to $30 \%$ of individuals with CF develop focal biliary cirrhosis, and at most 5 to $10 \%$ develop multilobular cirrhosis. Overall, biliary manifestations of CFLD are seen in 5 to $33 \%$ of patients and, besides dilatation or stricturization of the intra- and extrahepatic bile ducts and stone formation, they may include gallbladder abnormalities (micro gallbladder, gallbladder thickening and contraction, or gallstones). A significantly abnormal US has been shown to be a good predictor of advanced CFLD, with a specificity of approximately $84 \%$. Due to all these facts, annual US should be performed in all patients with $\mathrm{CF}$ as a valuable marker for detecting liver disease, assessing the severity and development of complications $(38,39,40,41)$.
Findings encompassing hepatomegaly, hyper-echogenicity (fatty infiltration), heterogeneous texture (fibrosis), with focal hyper- or hypoechoic lesions in children may be found in rare inborn errors of metabolism such as like glycogen storage disease (GSD), alpha 1 antitrypsin deficiency, tyrosinaemia type 1 or Gaucher disease (42). Some of these metabolic conditions are more prone to developing benign tumours (usually adenomas), or malignant ones, even in childhood (Fig. 2).

Studies have shown that between 16 to 75\% patients with GSD-I develop hepatic adenoma by puberty, while its prevalence increases with age. Moreover, adenomas had been reported to undergo malignant transformation in several cases, even in the absence of concomitant cirrhosis. For GSD III, adenomas are seen in 5 to $25 \%$ of patients; for GDS IV even less frequently (43).

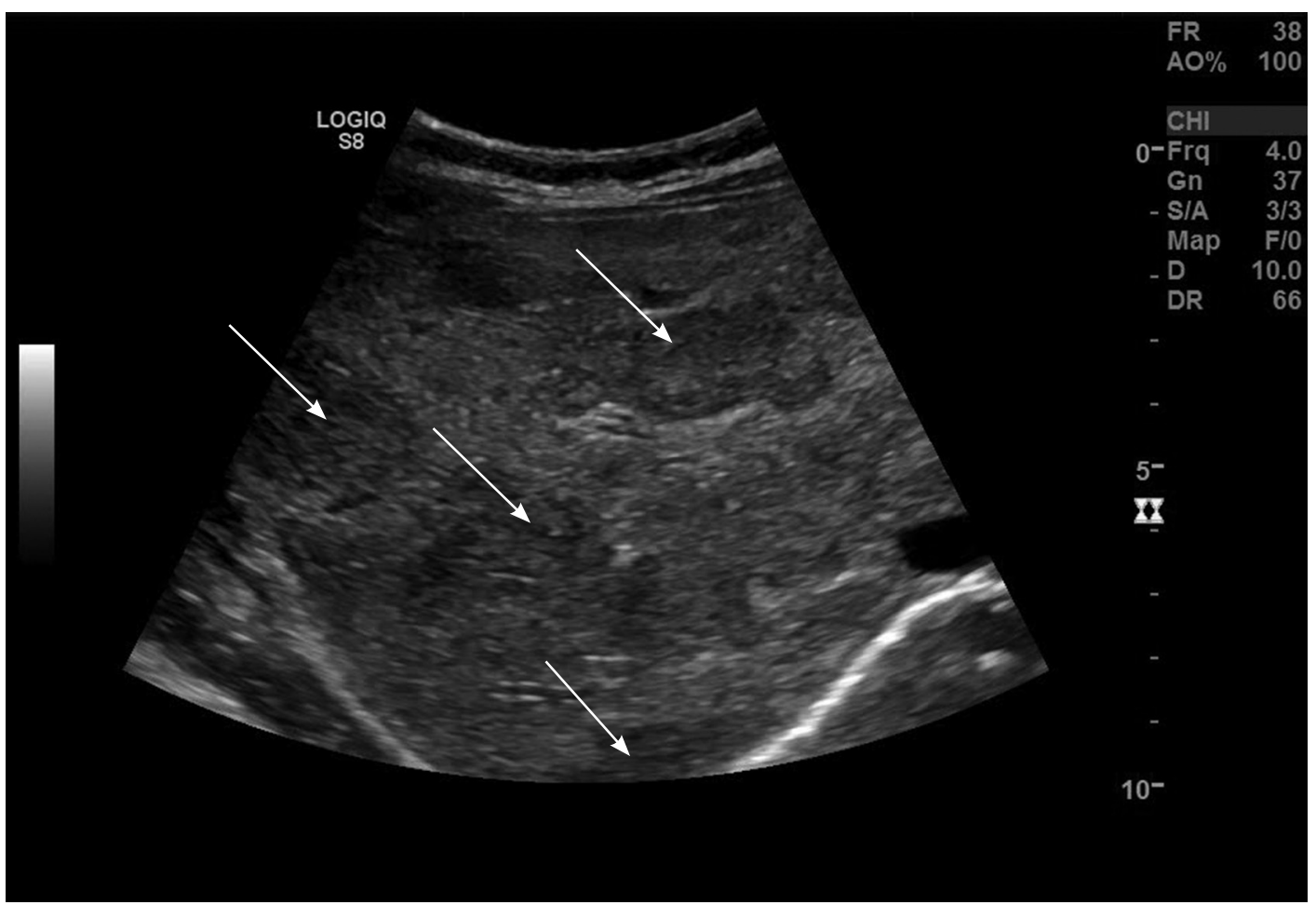

Fig. 2 Ultrasound of a patient with hepatic adenomatosis: several, different sized, predominantly hypoechoic masses in right liver lobe. 
Studies evaluating the incidence of HCC in CLD had shown increased frequency, not only in patients with inherited metabolic liver diseases (such as tyrosinemia type I), but also in chronic viral hepatitis. Although the mechanism of carcinogenesis remains to be fully understood, it is obvious that the liver may respond with hepatocellular proliferation to persistent stimulus in the CLD. US has proven to be an excellent screening and surveillance tool for all tumours in children with CLD. A recent expert viewpoint suggests that screening for HCC may improve its prognosis and is cost-effective, especially in patients with end-stage liver disease waiting for transplantation. On the basis of these data, it seems wise to follow up patients with CLD periodically by US. However, precise recommendations on 'how often' have still not been made (44).

\section{Liver biopsy}

For definitive diagnosing, staging and prognostic evaluation of the majority of CLDs, liver biopsy (LB) is an unavoidable procedure. Ultrasonographic scanning is mandatory for all patients before the LB is performed, to rule out anatomical anomalies, determine the best LB approach and lower the rate of post-biopsy complications. The majority of children are still subjected to 'blind LB', without simultaneous imaging study guidance. In order to further improve safety, and obtain high-quality tissue sample, a 'US-assisted LB' seems a more optimal variant. It includes ultrasonographic prebiopsy checking (immediately before the procedure) of the proposed biopsy site. The safest procedure is LB with ultrasonographic guidance, which further reduces complications. Hence, whenever possible, but always in cases defined as risky (very obese children, rare anatomical landmarks or possible focal lesions in the parenchyma), 'US-guided LB' should be used. Likewise, for cases of liver biopsy after segmental liver graft transplantation, 'US-guided' is a matter of choice. Postbiopsy complications (pain, bleeding, infection, haemobilia) have been reported in $1 \%$ of paediatric patients, with clinical symptoms occurring mostly within 4 hours. US at the bedside has a major role in detecting some of them, particularly bleeding, and should be required in case of any suspicion (45).

\section{Conclusion}

Conventional US still has a major role in the diagnosis and management of chronic liver diseases. Regardless of technological progress in diagnostic methodology, it remains indispensable for liver pathology. This is mainly due to the following advantages: its non-invasiveness, lack of radiation, availability, cost benefit, as well as providing significant diagnostic and prognostic information, detecting complications, and backing up LB. On the other hand, US cannot completely distinguish between fatty, fatty fibrotic or mildly fibrotic livers. Objective quantification of steatosis and its differentiation from NASH are also not possible. Likewise, a US finding is usually not specific enough for conclusive clarification of the aetiology of chronic liver diseases. Hence, the available evidence does not suggest the use of US as the sole method for the ultimate diagnosis in the majority of cases. It is better to interpret the results in the context of the whole clinical picture and, in inconclusive cases, it should be followed with additional imaging and laboratory work ups. Consequently, it should be the first, but not the last imaging modality for exploring the liver in daily clinical practice.

Authors' contributions: Conception and design: ACR, IP; Acquisition, analysis and interpretation of data: AJK, ACR, IP; Drafting the article AJK, IP; Revising it critically for important intellectual content: AJK, ACR, IP.

Conflict of interest: The authors declare that they have no conflict of interest. 


\section{References}

1. Vo NJ, Shet N. Radiology of the liver in children. In: Murray KF, Horslen S, editors. Diseases of the Liver in Children: Evaluation and Management. New York: Springer; 2013. p. 85-106.

2. Deganello A, Sellars MEK. Diagnostic procedures in pediatric hepatology. In: Guandalini S, Dahwan A, Branski, editors. Textbook of pediatric gastroenteorlogy, hepatology and nutrition. A comprehensive guide to practice. New York: Springer; 2015. p. 613-23.

3. Gubernick JA, Rosenberg HK, Ilaslan H, Kessler A. US Approach to jaundice in infants and children. Radigraphics. 2000;20(1):173-95.

4. De Bruyn R. The liver spleen and pancreas. In: De Bruyn R, editor. Pediatric Ultrasound: How, Why and When. London: Churchill Livingstone; 2010. p. 131-80.

5. Safak AA, Simsek E, Bahcebasi T. Sonographic Assessment of the Normal Limits and Percentile Curves of Liver, Spleen, and Kidney Dimensions in Healthy School-Aged Children. J Ultrasound Med. 2005;24:1359-64.

6. Boueva A. Ultrasound in Pediatrics. In: Tchacarski V, editor. Atlas of diagnostic Ultrasound: Ultrasonography. Sophia: Kindle; 2015, p.531-97.

7. Teele RL, Share JC. The liver. In: Teele RL, Share JC, editors. Ultrasonography of infants and children. Philadelphia: Saunders; 1991. p. 416-51.

8. Dietrich CF, Serra C, Jedrzejczyk K. Ultrasound of the liver. In: Dietrich CF, editor. EFSUMB European Course Book. London: European Federation of Societies for Ultrasound in Medicine and Biology; 2012. p. 31-90.

9. Tchelepi H, Ralls PW, Radin R, Grant E. Sonography of diffuse liver disease. J Ultrasound Med. 2002;21(9):1023-32.

10. Fitzpatrick E, Hadzic N. Paediatric non alcoholic fatty liver disease: An emerging treat. Paediatrics today. 2015;11:1-9.

11. DellaCorte C, Nobili V. Paediatric non alcoholic fatty liver disease: a growing problem. Paediatrics today. 2015;11(2):81-4.

12. Stal P. Liver fibrosis in non-alcoholic fatty liver disease - diagnostic challenge with prognostic significance. World J Gastroenterol. 2015;21(39):1107787.

13. Koplay M, Sivri M, Erdogan H, Nayman A. Importance of imaging and recent developments in diagnosis of nonalcoholic fatty liver disease. World J Hepatol. 2015;7(5):769-76.

14. Özcan HN, Oğuz B, Haliloğlu M, Orhan D, Karçaaltıncaba M. Imaging patterns of fatty liver in pediatric patients. Diagn Interv Radiol. 2015;21(4):355-60

15. Festi D, Schiumerini R, Marzi L, Di Biase AR, Mandolesi D, Montrone L, et al. Review article: the diagnosis of non-alcoholic fatty liver disease availability and accuracy of non-invasive methods. Aliment Pharmacol Ther. 2013;37(4):392-400.

16. Awai HI, Newton KP, Sirlin CB, Behling C, Schwimmer JB. Evidence and recommendations for imaging liver fat in children, based on systematic review. Clin Gastroenterol Hepatol. 2014;12(5):765-73.

17. Bohte AE, Koot BG, Van Der Baan-Slootweg OH, Van Werven JR, Bipat S, Nederveen AJ, et al. Us not be used to predict the presence or severity of hepatic steatosis in severely obese adolescents. Radiology. 2012;262(1):327-34.

18. Pinto RB, Schneider AC, da Silveira TR. Cirrhosis in children and adolescents: An overview. World J Hepatol. 2015;7(3):392-405.

19. Flores-Calderon J, Moran-Villota S, Ramon-Garcia G, Gonzalez-Romano B, Bojorquez-Ramos Mdel C, Cerdan-Silva L, et al. Non-invasive markers of liver fibrosis in chronic liver disease in a group of Mexican children. A multicenter study. Ann Hepatol. 2012;11(3):364-8.

20. Schuppan D, Afdhal NH. Liver cirrhosis. Lancet. 2008;371(9615):838-51.

21. Gerstenmaier JF, Gibson RN. Ultrasound in chronic liver disease. Insights Imaging. 2014;5:441-55.

22. Tutar O, Beser OF, Adaletli I, Tunc N, Gulcu D, Kantarci F, Mihmanli I, et al. Shear wave elastography in the evaluation of liver fibrosis in children. J Pediatr Gastroenterol Nutr. 2014;58(6):750-5.

23. Liszewski MC, Sleman IH. Ultrasound elastography in the noninvasive diagnosis of liver disease in children: a review. Paediatrics Today. 2016; 12(1):41-48.

24. Roberts EA. The jaundiced baby. In: Kelly DA, editor. Diseases of the liver and biliary system in Children. Oxford: Wiley - Blackwell: third edition; 2008, p. 57-105.

25. Zani-Ruttenstock E, Davenport M. Biliary atresia and choledochal malformation. In: Guandalini S, Dahwan A, Branski, editors. Textbook of pediatric 
gastroenteorlogy, hepatology and nutrition. A comprehensive guide to practice. New York: Springer; 2015. p. 633-45.

26. Millar AJW. Surgical disorder of the liver and bile ducts and portal hypertension. In: Kelly D, editor. Diseases of the liver and biliary system in Children. Oxford: Wiley - Blackwell: third edition; 2008. p. 433-74.

27. Bhavsar MS, Vora HB, Giriyappa VH. Choledochal cysts: a review of literature. Saudi J Gastroenterol. 2012;18(4):230-36.

28. Chen J, Tang Y, Wang Z, Wang Q, Wang D. Clinical value of ultrasound in diagnosing pediatric choledochal cyst perforation. AJR Am J Roentgenol. 2015;204(3):630-5.

29. Hee-Jung Lee, Sung-Moon Lee, Woo-Hyun Park, Soon-Ok Choi. Objective Criteria of Triangular Cord Sign in Biliary Atresia on US Scans. Radiology. 2003;229(2):395-400.

30. Jancelewicz T, Barmherzig R, Chung CT, Ling SC, Kamath BM, Ng VL, at al. A screening algorithm for the efficient exclusion of biliary atresia in infants with cholestatic jaundice. J Pediatr Surg. 2015;50(3):363-70.

31. Jiang LP, Chen YC, Ding L, Liu XL, Li KY, Huang $\mathrm{DZ}$, at al. The diagnostic value of high-frequency ultrasonography in biliary atresia. Hepatobiliary Pancreat Dis Int. 2013;12(4):415-22.

32. Kelly D. The Approach to the Child with Liver Disease: Differential Diagnosis and Useful Investigations In: Kelly D, editor. Diseases of the liver and biliary system in Children. Oxford: Wiley- Blackwel: third edition; 2008. p. 21-34.

33. Soresi M, Bonfissuto G, Magliarisi C, Riili A, Terranova A, Di Giovanni G, et al. Ultrasound detection of abdominal lymph nodes in chronic liver diseases. A retrospective analysis. Clin Radiol. 2003;58(5):372-7.

34. Mieli-Vergani G, Vergani D. Autoimmune liver disease. In: Guandalini S, Dahwan A, Branski, editors. Textbook of pediatric gastroenteorlogy, hepatology and nutrition. A comprehensive guide to practice. New York: Springer; 2015. p. 705-16.
35. Feldstein AE, Perrault J, El-Youssif M, Lindor KD, Freese DK, Angulo P. Primary sclerosing cholangitis in children: a long-term follow-up study. Hepatology. 2003;38(1):210-7.

36. Akhan O, Akpinar E, Karcaaltincaba M, Haliloglu M, Akata D, Karaosmanoglu AD, et al. Imaging findings of liver involvement of Wilson's disease. Eur J Radiol. 2009;69(1):147-55.

37. European Association for Study of Liver EASL. Clinical Practice Guidelines: Wilson's disease. J Hepatol. 2012;56(3):671-85.

38. Parisi GF, Di Dio G, Franzonello C, Gennaro A, Rotolo N, Lionetti E, at al. Liver disease in cystic fibrosis: an update. Hepat Mon. 2013;13(8):e11215.

39. Wilchanski M, Uc A. Cystic fibrosis. In: Guandalini S, Dahwan A, Branski, editors. Textbook of pediatric gastroenteorlogy, hepatology and nutrition. A comprehensive guide to practice. New York: Springer; 2015. p. 471-85.

40. Flass T, Narkewicz MR. Cirrhosis and other liver disease in cystic fibrosis. J Cyst Fibros. 2013;12(2):116-24

41. Leung DH, Ye W, Molleston JP, Weymann A, Ling $S$, Paranjape SM, et al. Baseline Ultrasound and Clinical Correlates in Children with Cystic Fibrosis. J Pediatr. 2015;167(4):862-8.

42. Morgan T, Qayyum A, Gore MR. Diffuse liver disease. In: Gore MR, Levine MS, editors. Textbook of Gastrointestinal Radiology: Philadelphia: Elselvier Sounders: fourth edition; 2015. p. 1629-75.

43. Shady DA, Roy A, Finegold A. Liver tumors in children with metabolic disorders. Transl Pediatr. 2015;4(4):290-303.

44. Rosetnthal P. Hepatocarcinoma in Viral and Metabolic Liver Disease. J Pediatr Gastroenterol Nutr. 2008;46(4):370-5.

45. Dezsőfi A, Baumann U, Dhawan A, Durmaz O, Fischler B, Hadzic N, et. al. Liver biopsy in children: position paper of the ESPGHAN Hepatology Committee. J Pediatr Gastroenterol Nutr. 2015;60(3):408-20. 